\title{
Remediation and by-products of MTBE in groundwater by a photochemical process
}

\author{
S. Mohebali, S. Tasharrofi \& R. Kaveh Ahangar \\ Industrial \& Environmental Protection Div., \\ Research Institute of Petroleum Industry, Iran
}

\begin{abstract}
MTBE has been used as an octane number enhancer in gasoline, and blended with gasoline to about $15 \%$ by volume. High concentrations of MTBE have been discovered in the groundwater at a number of petrol stations in Iran. MTBE exhibits very high mobility in groundwater. Due to its solubility, groundwater contaminated with MTBE poses many problems for remediation. The properties of MTBE, including water solubility, vapour pressure, soil adsorption coefficient, retardation factor and Henry's law constant affect the selection and design of remediation technologies. Although MTBE is difficult to treat economically with conventional techniques, it readily degraded photocatalytically. The degradation of MTBE at high concentration $(10,000 \mu \mathrm{g} / \mathrm{l})$ was investigated in batch nanocrystalline $\mathrm{TiO}_{2}$ slurries. The optimum loading of $\mathrm{TiO}_{2}$ was found experimentally. In this experiment MTBE degradation proceeded with a pseudo first order reaction. The primary by-products have been identified. Mass balance calculation has shown that MTBE is completely mineralised. A reaction pathway is proposed.
\end{abstract}

Keywords: photocatalysis, advanced oxidation process, nanocrystalline titanium dioxide, hydroxyl radical, tert-butyl format, tert-butyl alcohol, MTBE.

\section{Introduction}

MTBE has been widely used as an octane enhancer in gasoline in Iran since 2000. The gasoline has been stored in aboveground and underground storage tanks at refineries and service stations, and has been transferred via pipeline, barge, rail and truck. As a result of leaks and splits, MTBE has been detected in groundwater supplies more than $2 \mu \mathrm{g} / 1$ [1]. The physical properties of MTBE 
including its solubility in water and volatility from the free product phase cause a big mobility in the environment. Due to MTBE's physical properties, it has been difficult to treat contaminated groundwater with conventional methods. MTBE is approximately 10 times less volatile than benzene making air stripping less efficient. Also MTBE does not adsorb well to granular activated carbon (GAC) compared to benzene, GAC is about five times less effective at removing MTBE $[2,3]$. Two bio recalcitrant functional groups in MTBE structure, ether link and branched, make it resistant to biodegradation. Since bioremediation strategies are often used to degrade petroleum hydrocarbons, it would be advantageous to either enhance the biodegradability of MTBE or to find a destruction process, which could be combining well with bioremediation.

Advanced oxidation processes (AOPs), which involve the generation of the hydroxyl radical $(\mathrm{OH})$ to degrade a chemical, have recently emerged as an important class of technologies for accelerating the oxidation and destruction of a wide range of organic contaminants in polluted water and soil. An advantage of AOPs over activated carbon and air stripping is that, if effective, the contaminant is degraded into other compounds, removing the contaminant from the environment, not just from the aqueous phase.

In this research, photocatalytic degradation of MTBE in nanocrystalline $\mathrm{TiO}_{2}$ slurry was investigated by (i) quantifying the kinetics of MTBE degradation, (ii) identifying the by-products formed, (iii) proposing a reaction scheme.

\section{Materials and methods}

MTBE (Merck), t-butyl format (TBF, Aldrich) were reagent grade. Nanocrystalline Titanium dioxide $\left(\mathrm{TiO}_{2}\right)$ was obtained from Degussa corp. (p25).

Photochemical experiments were carried out in a $700 \mathrm{ml}$ batch slurry photoreactor with a UV lamp immersed in the centre of photoreactor. Slurry was circulated with a pump through the reactor in a constant rate. A reaction run involved the addition of $\mathrm{TiO}_{2} 700 \mathrm{ml}$ of deionised water and $10 \mu \mathrm{l}$ of MTBE $(10750 \mu \mathrm{g} / \mathrm{l})$ to the reactor. $\mathrm{TiO}_{2}$ particles were allowed to be settling down overnight in $4{ }^{\circ} \mathrm{C}$ [4].

Reaction kinetics was followed using gas chromatography (GC). Compounds were extracted from the aqueous solution with a Lekmar ISC 200 purge and trap apparatus, with conditions as specified in EPA methods 8015, 5030. The trapped compounds were auto-injected into a Varian model 3400 gas chromatograph with flame ionisation detector (FID). CP.CIL5, $60 \mathrm{~m}, 0.75$ i.d. capillary columns were used with the following temperature program; initial temperature $150{ }^{\circ} \mathrm{C}$, rate of increasing temperature $5{ }^{\circ} \mathrm{C} / \mathrm{min}$, final temperature $200{ }^{\circ} \mathrm{C}$. Unknown reaction by-products were identified by Head-Space gas chromatography-mass spectrometry (HS/GC-MS). Chromatographic analysis was performed using a Varian model $1200 \mathrm{GC}$ equipped with a Quadrupole mass spectrometer. Samples were introduced into the GC with HS system. Separation of the analytes was performed using a $60 \mathrm{~m} \times 0.25 \mu \mathrm{m}$ I.D. VF5 column with a film thickness of $0.25 \mu \mathrm{m}$. The column oven temperature was initially at $50{ }^{\circ} \mathrm{C}$ Temperature 
increased at a rate of $5{ }^{\circ} \mathrm{C} / \mathrm{min}$ to $100^{\circ} \mathrm{C}$ and then at a rate of $20^{\circ} \mathrm{C}$ to final temperature $275^{\circ} \mathrm{C}$. Mass spectra were scanned in the range of $32-450 \mathrm{~m} / \mathrm{e}$. identification of the individual compounds was made by comparing the resulting spectral pattern with those of the information service spectra library.

Soluble organic acids were analysed using a metrohm modular Ion chromatograph with a metrosep A SUPP 5-250 column and conductivity detector. The eluent for the process was a $1 \mathrm{mmole} / 1$ solution of $\mathrm{NaHCO}_{3}$ and 3.2 mmole/l solution of $\mathrm{Na}_{2} \mathrm{CO}_{3}$ with a flow rate of $0.7 \mathrm{ml} / \mathrm{min}$. Peak identification was performed by peak matching using $1 \mathrm{mg} / \mathrm{l}$ solution of acetic acid and formic acid.

\section{Result and discussion}

A series of experiment was carried out to find an optimum catalyst loading. By varying the amount of $\mathrm{TiO}_{2}$ in the reaction from $0.01 \mathrm{~g}$ to $0.3 \mathrm{~g}$. it was found that the optimum loading of catalyst was $0.05 \mathrm{~g}$ per $700 \mathrm{ml}$ of solution $(0.07 \mathrm{~g} / \mathrm{l})$. Above this amount increased turbidity of the solution reduced the light transmission through the solution. Below this amount, catalyst surface was limiting and photocatalytic efficiency decreased. Photocatalytic degradation of MTBE at this optimum loading is shown in Fig. 1. It was observed that more than $80 \%$ of MTBE was removed in the first 20 min of the reaction. Nearly complete removal of MTBE ( $>99.99 \%)$ was achieved after $120 \mathrm{~min}$. A first order model according to Fig. 2 with the rate constant of $6.4 \times 10^{-2} \mathrm{~min}^{-1}$ estimated the degradation rate constant. After $2 \mathrm{~h}$ MTBE was no longer detectable in the reaction solution.

By-products of photocatalytic degradation of MTBE were identified by $\mathrm{HS} / \mathrm{GC} / \mathrm{MS}$. As shown in fig. 3, seven components exist in the chromatogram. By comparing the spectrum of each component (fig 4-8) with the standard spectrum of instrument service spectra library, five by products were identified.

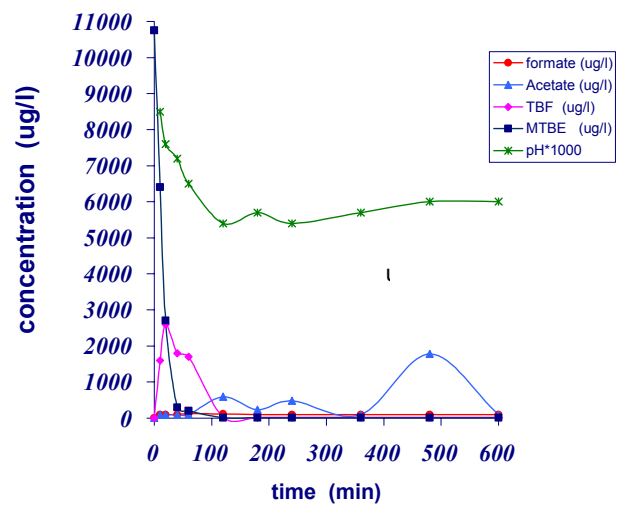

Figure 1: The variation with time of the concentration of MTBE and its byproducts and the $\mathrm{pH}$ solution. 


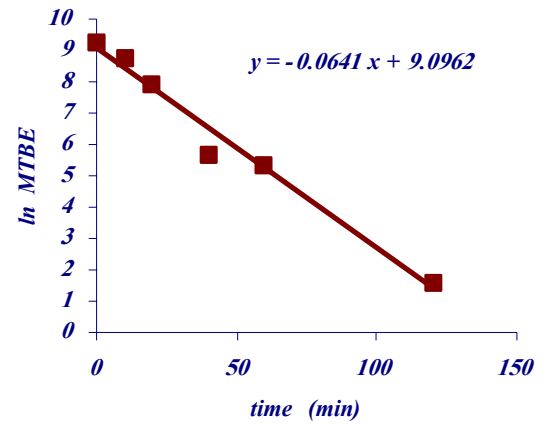

Figure 2: The photocatalytic reaction rate of MTBE.

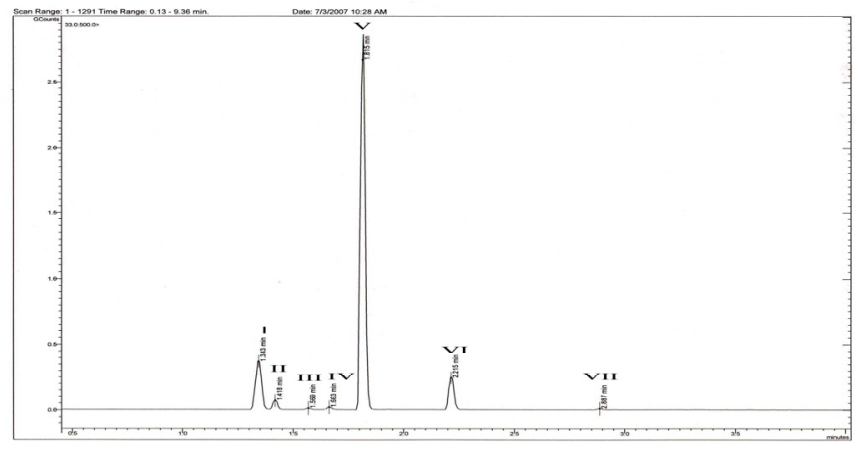

Figure 3: Chromatogram of reaction mixture; I) CO2, II) 2-methyl-1propene, III) acetone, IV) TBA, V) MTBE, VI) TBF, VII) 2methyl propanoic acid.

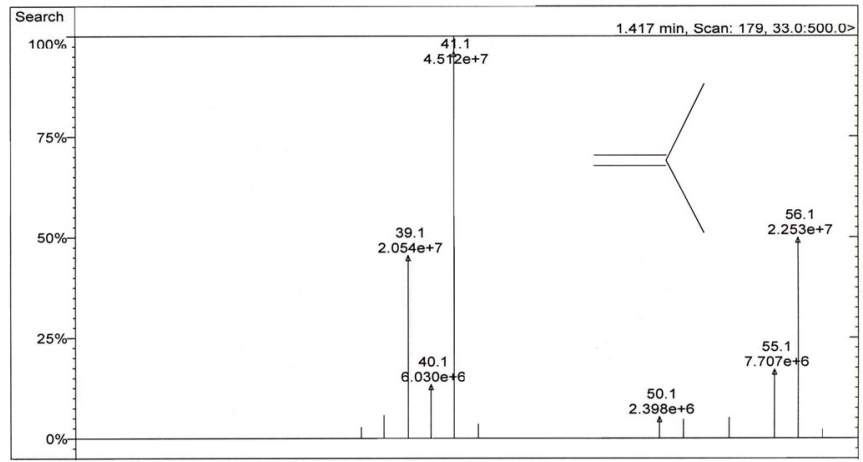

Figure 4: $\quad$ Mass spectrum of 2-methyl-1-propene. 


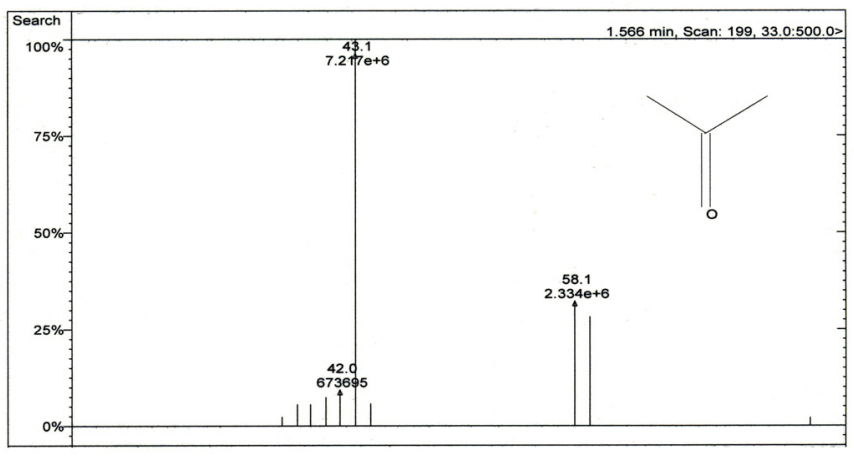

Figure 5: $\quad$ Mass spectrum of acetone.

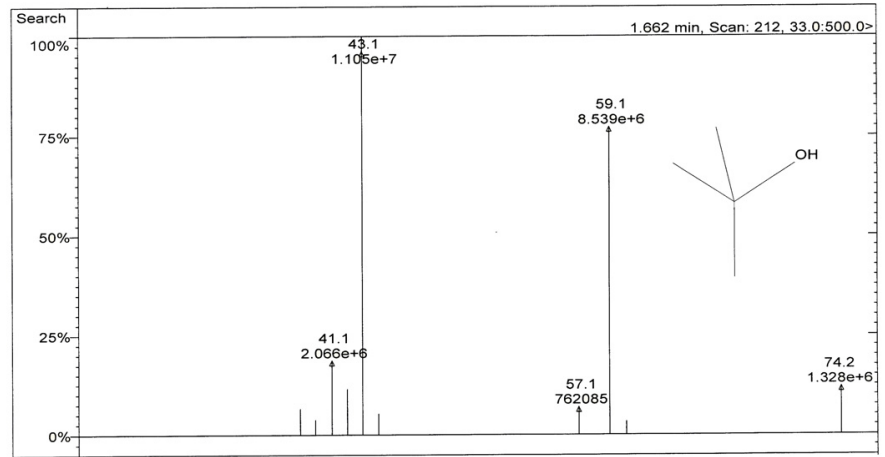

Figure 6: $\quad$ Mass spectrum of TBA.

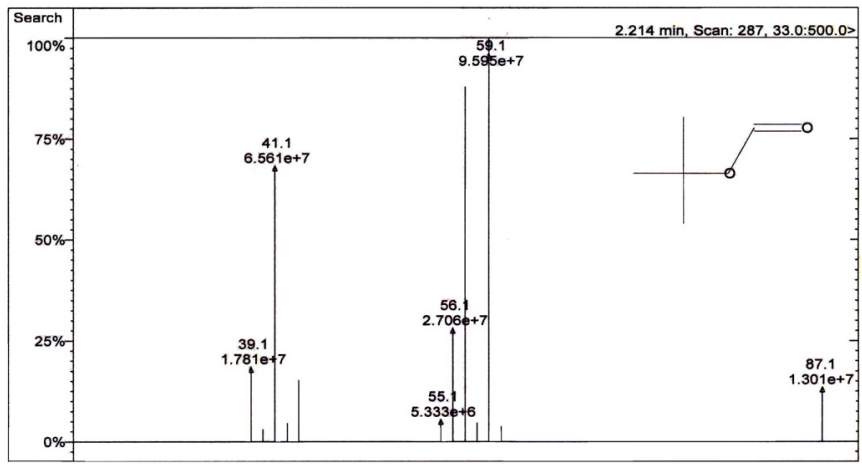

Figure 7: $\quad$ Mass spectrum of TBF.

Two peaks in 1.318, 1.815 min related to dissolved carbon dioxide and MTBE respectively. The major by-products were t-butyl format (TBF), t-butyl alcohol (TBA), and acetone. Two other by-products were 2-methyl-1-propene and 
isopropanoic acid. TBF is the first by-product of degradation with higher toxicity than MTBE. It appeared rapidly and reached to a maximum at $20 \mathrm{~min}$. However TBF is also mineralised and after $3 \mathrm{~h}$ the concentration of TBF decreased to a trace level.

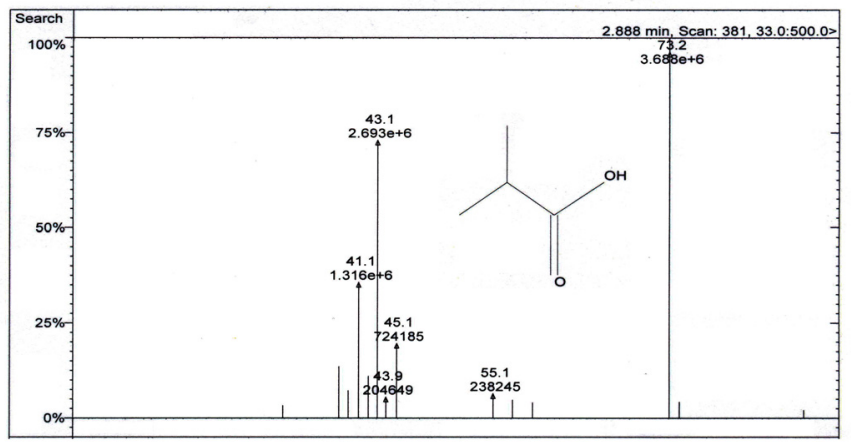

Figure 8: $\quad$ Mass spectrum of 2-methyl propanoic acid.

The decreasing of $\mathrm{pH}$ during the reaction suggested the presence of acidic compounds. Ion chromatography showed the presence of two organic acid acetic acid and formic acid were identified by retention time matching with external standards. The formation acidic product was consistent with $\mathrm{pH}$ changes. The initial $\mathrm{pH}$ of solution was 8.5 and gradually decreased. After $1 \mathrm{~h}$ of reaction the $\mathrm{pH}$ dropped to 6.5 due to formation of formic acid. Over the reminder of the degradation the $\mathrm{pH}$ slowly dropped to 5.4 because of appearance of acetic acid. However the $\mathrm{pH}$ reached to 6 after $8 \mathrm{~h}$ and no $\mathrm{pH}$ changes was detected in control reaction. At $10 \mathrm{~h}$ of reaction no organic products were detected. A mass balance on organic carbon was determined for the reaction solution at $10 \mathrm{~h}$ and approximately $99.9 \%$ of MTBE was mineralised to $\mathrm{CO}_{2}$ and water. According to above information, a reaction scheme was proposed (Fig 9).

It is well accepted that the initial process for photocatalysis of organic compounds by semiconductors is the generation of electron-hole pairs in the $\mathrm{TiO}_{2}$ particles $[5,6]$.

$$
\mathrm{TiO}_{2} \stackrel{h r}{\longrightarrow} \bar{e}+h^{+}
$$

$\mathrm{TiO}_{2}$ is a semiconductor with a band gap of $3.2 \mathrm{eV}$; when excited by light of energy equal to or exceeding that value, electrons are promoted from the valance band to the conduction band, leaving positive holes in the valance bands. These electrons and holes are capable of reducing and oxidizing compounds at the $\mathrm{TiO}_{2}$ surface. The hole is quickly converted to the hydroxyl radical $(\mathrm{OH})$ upon oxidation of surface adsorbed water. This radical is the major reactant responsible for the oxidation of organics. The first step in the destruction of MTBE is abstraction of an $\alpha$-hydrogen by hydroxyl radical to form an organic radical. This radical reacts with dissolved oxygen in the reaction solution to convert a proxy radical as an unstable intermediate. The most important oxidation of ethers is conversion to esters. Therefore the unstable intermediate 


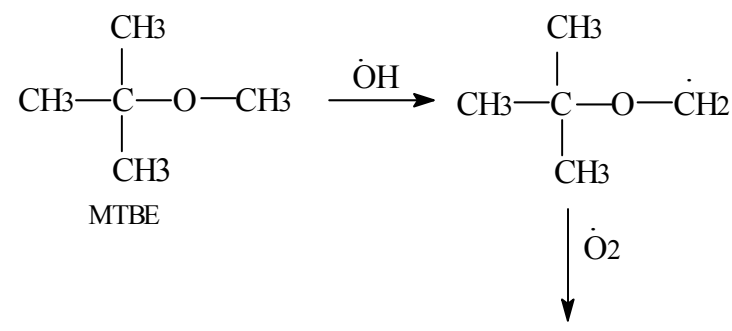

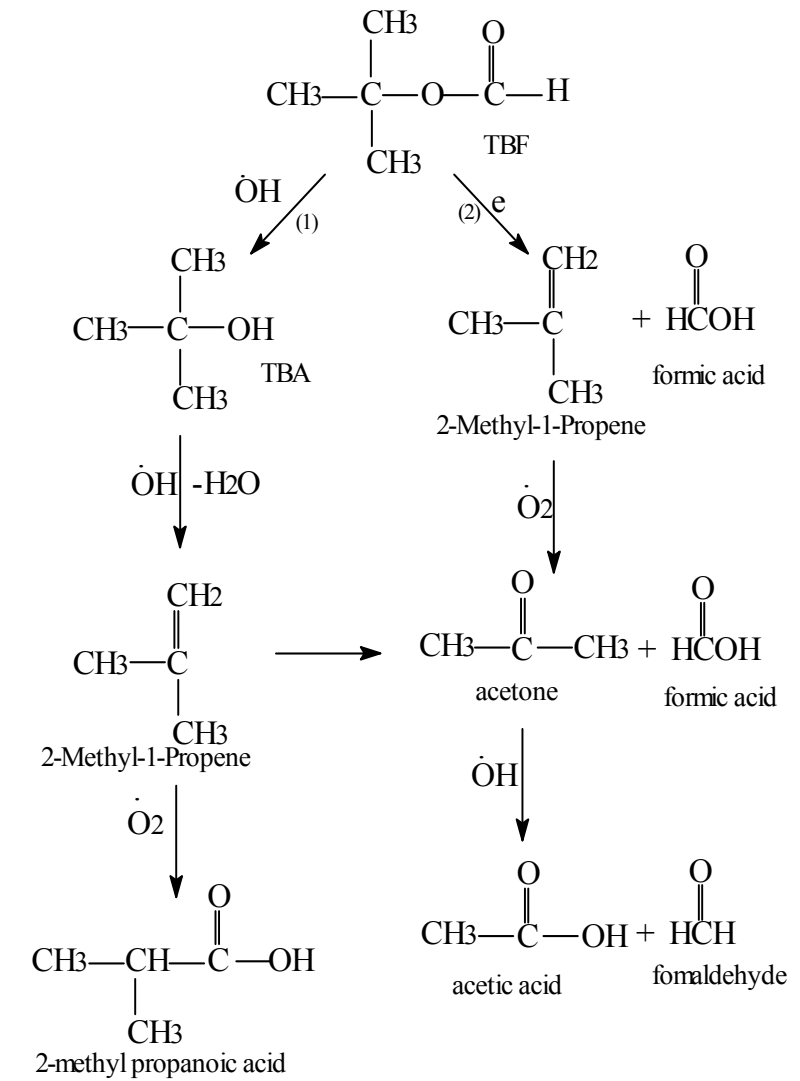

Figure 9: Proposed reaction pathway for photocatalytic destruction of MTBE.

rapidly was converted to TBF by heterolysis of the O-O band. Although hydroxyl radicals are strong oxidants in this reaction, other active species such as electron and super oxide anions can react with organic compounds. Due to this fact, TBF degraded in two ways. Esters are subject to both acid and base catalysed hydrolysis to produce carboxylic acid and alcohol. Therefore in the first way, TBF would produce TBA and formic acid. Formic acid is photocatalytically oxidized by oxygen to $\mathrm{CO}_{2}$ and water on the surface of $\mathrm{TiO}_{2}$. 
In the second way, TBF reduced with electron to form 2-methyl-1-propene and formic acid. Under acidic condition and in the presence of hydroxyl radicals, TBA was dehydrated to alkenes, which easily oxidized and produced acetone and formic acid. Then acetic acid, like other by-product, was photocatalytically degraded to acetic acid and formaldehyde. When the time of reaction proceeded, all organics was degraded to $\mathrm{CO}_{2}$ and water.

The degradation of TBF was a first order reaction with a rate faster than MTBE. The stoichiometry of reaction confirmed this fact, because the concentration of $\mathrm{TBF}$ at $10 \mathrm{~min}$ in the reaction solution was not equal to the concentration of destruction MTBE. Also TBF disappeared after $3 \mathrm{~h}$.

Investigation of the concentration of acetic acid is useful to predict the rate of degradation of TBA. As shown in Fig.1, there are a maximum at the curve for acetic acid at $6 \mathrm{~h}$ that relate to degradation of TBA to acetone and then to acetic acid. It means the rate of degradation of TBA is slower than MTBE and TBF and this step was the limiting step in the photocatalytic process.

\section{Conclusion}

It has been found that MTBE is readily and rapidly degraded photocatalytically by nanocrystalline $\mathrm{TiO}_{2}$ under batch slurry conditions with first order rate constant of $6.4 \times 10^{-2} \mathrm{~min}^{-1}$. The primary intermediates of this reaction have been identified to be t-butyl format, t-butyl alcohol and acetone, which are easily degraded photocatalytically. TBF is degraded faster and TBA slower than MTBE. A reaction scheme has been proposed by considering the action of active species generated when $\mathrm{TiO}_{2}$ excited by UV radiation. MTBE completely degraded to biodegradable products. Upon which to consider using photocatalysis reaction as a method to convert a non-biodegradable compound such as MTBE and it's the first by-product, TBF, to products such as TBA and acetone which can be treated biologically [7].

\section{References}

[1] K. Torkestani, et al, "determination of MTBE in groundwater near gasoline station and refinery”, A Project Report for RIPI, No. 1248-281, 2006.

[2] EPA 510-F-97-015, Jan 1998.

[3] N.E. Kinner, "Fate, Transport and Remediation of MTBE “, A Report for University of New Hampshire, April 2001.

[4] R.D. Barreto, K.A. Gary, water research; Vol.29, No.5, pp.1243-1248.1995.

[5] A. Asadi, M. Mehrvar, International journal of photoenergy, Article ID 19790, 2006.

[6] H. Gnaser, B.C. Zeigler," encyclopedia of nanoscience and nanotechnology", (Ed.H.S.Nalwa), Vol.6, pp. 505-535, 2004.

[7] S. Esplugas, A. Marco, "How and Why Combine Chemical and Biological Processes for Wastewater Treatment", A Report for Chemical Engineering and Metallurgy University. 